\title{
Efficient Computation of Current Collection in Bare Electrodynamic Tethers in and beyond OML Regime
}

\author{
M. Sanjurjo-Rivo ${ }^{1}$; G. Sánchez-Arriaga ${ }^{2}$; and J. Peláez ${ }^{3}$
}

\begin{abstract}
One key issue in the simulation of bare electrodynamic tethers (EDTs) is the accurate and fast computation of the collected current, an ambient dependent operation necessary to determine the Lorentz force for each time step. This paper introduces a novel semianalytical solution that allows researchers to compute the current distribution along the tether efficient and effectively under orbital-motion-limited (OML) and beyond OML conditions, i.e., if tether radius is greater than a certain ambient dependent threshold. The method reduces the original boundary value problem to a couple of nonlinear equations. If certain dimensionless variables are used, the beyond OML effect just makes the tether characteristic length $L^{*}$ larger and it is decoupled from the current determination problem. A validation of the results and a comparison of the performance in terms of the time consumed is provided, with respect to a previous ad hoc solution and a conventional shooting method. DOI: 10.1061/(ASCE)AS.1943-5525.0000479. @ 2014 American Society of Civil Engineers.
\end{abstract}

\section{Introduction}

The use of electrodynamic tethers (EDTs) has been proposed as an alternative and efficient solution in scenarios, such as orbital debris mitigation (Ahedo and Sanmartín 2002; Johnson et al. 2000; Peláez and Sanjurjo 2006) and planetary exploration (Sanmartín and Lorenzini 2005). An analysis of both applications can be found in Sanmartín et al. (2010) and Sánchez-Torres (2013). Electrodynamic tethers are able to provide thrust or generate electric power by converting from electrical to mechanical energy of the tethered system, depending on the operating regime. There are two different regimes, as follows: (1) active regime, which corresponds in general with the former case; and (2) passive regime, which corresponds in general with the latter case. In both cases, the propellant mass consumption is small compared to other propulsion systems (Sanmartin et al. 2006b).

The concept of bare EDTs was presented for the first time in Sanmartín et al. (1993). The formulation of the current profile computation problem, a necessary issue to find the Lorentz force was also posed as well as the operation boundaries in the orbitalmotion-limited (OML) regime (Sanmartín and Estes 1999). In the seminal and subsequent articles (Ahedo and Sanmartín 2002; Sanmartín et al. 2006a), a handful of analytical approximations and exact solutions were proposed, for different operational conditions and functions of EDTs operating under OML conditions.

The problem of obtaining the current profile along the tether was tackled by Leamy et al. (2001). Although the boundary conditions considered in that paper are different from those in this paper, the fundamentals for the resolution of the boundary value problem are similar. The system of differential equations with boundary conditions is turned into a set of algebraic nonlinear equations. This transformation can be carried out by means of quadratures that link the independent variable, the length along the tether, and the state variables (current $I$ and bias voltage $\Phi$ ). In this manner, functions $I$ and $\Phi$ can be described in terms of a single parameter. The previously mentioned mentioned relation between length and the variables of the problem entails the use of hypergeometric functions. Due to this, henceforth, this approach will be called the hypergeometric solution or formulation.

Recent results on tether mission design (J. R. Sanmartín et al., "Optimization method in tape tether sizing in de-orbiting satellites at the end of mission," submitted, ETSIA, Madrid, Spain), based on a tether survivability model (Khan and Sanmartin 2013), have shown that for certain missions, tethers with a high cross section can be useful. This can affect the collected current if tether radius $R$ (or width) is greater than a certain maximum $R_{\max }$, which depends on environmental conditions and tether parameters (Sanmartín and Estes 1999). For $R>R_{\max }$ the tether is said to operate beyond the OML regime and the OML current must be corrected (Estes and Sanmartín 2000) by a factor, say $G$, below unity. In this paper we were in accordance with the procedure introduced in G. SanchezArriaga et al. ("Impact of non ideal effects on bare electrodynamic tether performance," submitted, ETSIA, Madrid, Spain), which decouples the beyond OML effect from the determination of the current and potential profiles thanks to a rescaling of the dimensionless variables by the factor $G$. However, since these calculations must be done each time step along the tether flight simulation, the computation of $G$ with the algorithm described in Estes and Sanmartín (2000) may be computationally expensive. This issue is avoided in this paper by presenting an analytical fitting of the factor $G$ in a broad range of parameters.

This paper introduces a novel semianalytical solution of the current collection model. The approach is similar to the one described in Leamy et al. (2001). Nevertheless, it represents a further simplification of the solution and provides a faster computation of the current profile, as will be shown in this paper. This new formulation together with the incorporation of the beyond OML effect through an analytical fitting of the factor $G$ yields an efficient and accurate algorithm appropriate for accurate tether flight simulators. The validation of the semianalytical solution is made by comparing the results to Leamy et al. (2001) and a standard shooting method 
(e.g., Press et al. 1992, Chapter 18). The performance of the three methods is also compared.

\section{Operation of Electrodynamic Tethers}

Consider a rigid bare tether of length $L$, conductivity $\sigma$, and crosssectional area $A_{t}$. At one of its ends, named Point $\mathrm{C}$, it has a load of resistance $r$ or a battery that supplies an electromotive force, $\epsilon$, and next is a plasma contactor device (a hollow cathode or a thermionic emitter device), which ejects electrons at a cost of a potential drop $V_{c c}$ (Sanmartín et al. 1993). The opposite end, Point A, is the origin of a system of coordinates $S$ with its $x$-axis along the tether (Fig. 1). Two possible operation regimes are possible [i.e., (1) passive, and (2) active; Fig. 1]. For a detailed discussion on the differences between both regimes, refer to Sanmartín et al. (1993). In this paper, only the passive regime is addressed, although the methodology can be extended to the active regime without complication.

Thanks to the good and steady electrical contact between the tether and the surrounding ionospheric plasma, an electric current $\mathbf{I}=-I(x) \mathbf{u}_{x}$ flows along the tether. Its interaction with the ambient magnetic field $\mathbf{B}_{0}$ yields the Lorentz force

$$
\mathbf{F}=\int_{0}^{L} I(x) \mathbf{B}_{0} \times \mathbf{u}_{x} d x
$$

Current exchange between the plasma and the tether happens at the plasma contactor, and at the bare tether itself, thus acting as a very long Langmuir probe (Sanmartín et al. 1993). Plasma probe theory (Laframboise and Parker 1973) indicates that current collection is controlled by the local potential bias $\Phi(x)=V_{t}-V_{p l}$, where $V_{t}$ and $V_{p l}$ are the tether and faraway plasma potentials, respectively. Tether points within the range $0<x<L_{B}$ (anodic segment), where $\Phi(x)>0$, collect electrons. The current per unit length is (Laframboise and Parker 1973; Sanmartín et al. 1993; G. Sanchez-Arriaga et al., "Impact of non ideal effects on bare electrodynamic tether performance," submitted, ETSIA, Madrid, Spain)

$$
\frac{d I(x)}{d x}=G\left[\frac{e \Phi(x)}{k T_{i}} ; T_{e} / T_{i}, R / \lambda_{D i}\right] \times\left[e N_{0} \frac{p_{t}}{\pi} \sqrt{\frac{2 e \Phi(x)}{m_{e}}}\right]
$$

where $e=$ electron charge; $m_{\alpha}, T_{\alpha}$, and $\lambda_{\alpha}=$ mass, temperature, and Debye length (subscript $\alpha=e, i$ denotes electrons and ions);

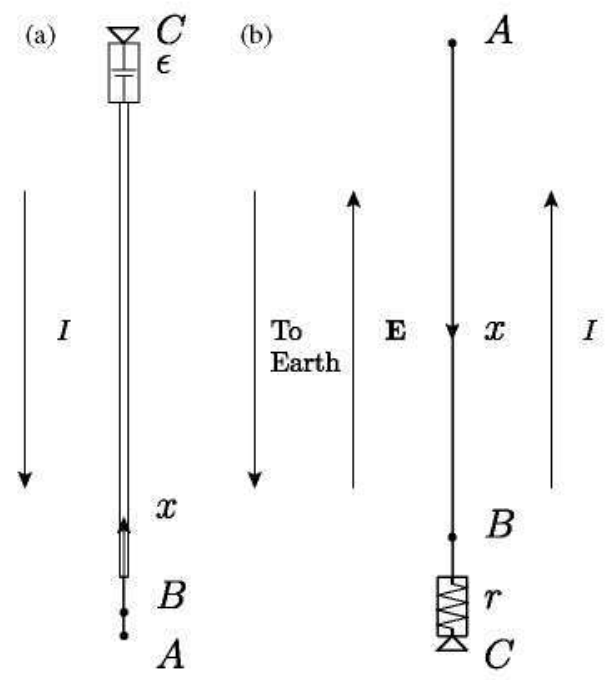

Fig. 1. Tether configurations in Earth prograde orbit: (a) active configuration; (b) passive configuration
$N_{0}=$ density of the ionospheric plasma; and $p_{t}$ and $R=$ perimeter and the radius of the tether $[R \approx w / 4$ if it is a tape with width equal to $w$ (Sanmartín and Estes 1999)]. The factor $G$ is a positive number below unity and it takes into account the (possible) operation of the tether beyond the so-called OML regime (Estes and Sanmartín 2000); see the Appendix for further details.

For tether points in the range $L_{B}<x<L$ (cathodic segment) with $\Phi(x)<0$, the current variation due to ion collection is

$$
\frac{d I(x)}{d x}=-G\left[\frac{e|\Phi(x)|}{k T_{e}} ; T_{i} / T_{e}, R / \lambda_{D e}\right] \times\left[e N_{0} \frac{p_{t}}{\pi} \sqrt{\frac{2 e(-\Phi)}{m_{i}}}\right]
$$

In this paper, it was considered that a hollow cathode operates at the cathodic end of the tether. With this arrangement, the cathodic segment is much shorter due to a very low contact impedance of the hollow cathode. Therefore, the current variation due to secondary emission of electrons in the cathodic region has been ignored. In general, this effect represents a small correction with respect to Eq. (3) and disregarding it, it is possible to obtain the semianalytical solution presented hereafter. This effect is nevertheless taken into account in previous studies (Sanmartin et al. 2006b) for situations in which the secondary emission plays a role.

The same function $G$ but with different arguments is used in Eqs. (2) and (3). Such a simple universal function, valid for both polarizations, would not be possible if any additional effect depending on the mass of the species is added. Two examples are (1) magnetic field effects, and (2) a plasma velocity relative to the probe, which introduce the Larmor radius and the ion (ram) energy, respectively.

Both $V_{t}$ and $V_{p l}$ vary along tether length. Current $I(x)$ and potential inside the tether $V_{t}$ satisfy Ohm's law $d V_{t} / d x=I(x) /\left(\sigma A_{t}\right)$. Regarding the faraway plasma potential, a motional electric field $\mathbf{E}=\mathbf{v}_{\mathrm{rel}} \times \mathbf{B}_{0}$ appears in the tether frame due to the tether-toplasma relative velocity $\mathbf{v}_{\text {rel }}$ and $\mathbf{B}_{0}$. Defining the projection of this field along the current direction, $E_{m}=-\mathbf{E} \cdot \mathbf{u}_{x}$, one finds $d V_{p l} / d x=E_{m}$. The equation for the local potential bias is

$$
\frac{d \Phi}{d x}=\frac{I(x)}{\sigma A_{t}}-E_{m}
$$

In the passive tether regime, the problem is closed by the circuit equation, which is obtained by integrating Eq. (4) between $L_{B}$ and $L$

$$
V_{c c}+r I_{C}=\left[E_{m}\left(L-L_{B}\right)\right]-\int_{L_{B}}^{L} \frac{I(x)}{\sigma A_{t}} d x
$$

with $I_{C} \equiv I(L)$, the current at the hollow cathode. Eqs. (2) and (5) together with the boundary conditions $I(0)=0, \Phi\left(L_{B}\right)=0$ gives the current and potential profiles $I(x)$ and $\Phi(x)$ together with the anodic length $L_{B}$.

\section{Current and Bias Differential Equations}

For convenience, nondimensional variables are used to state the ordinary differential equations for current and bias. The characteristic magnitudes that appear in the problem were already identified in Sanmartín et al. (1993). Lately, a new approach has been proposed using slightly different characteristic magnitudes (Bombardelli et al. 2010). The research reported in this paper has been carried out using a version of the former, modified to include the effect of operating beyond the OML regime. Thus, the characteristic length is $L^{*}$ (Sanmartín et al. 2001) 


$$
L^{*} \equiv\left[\frac{9 \pi^{2}}{128} \times \frac{1}{G^{2}\left(\beta_{i}, T_{e} / T_{i}, R / \lambda_{D i}\right)} \times \frac{m_{e} \sigma^{2}}{e^{3}} \times \frac{E_{m} h^{2}}{N_{0}^{2}}\right]^{1 / 3}
$$

where $\beta_{\alpha} \equiv\left(e E_{m} L^{*}\right) /\left(k T_{\alpha}\right)$ and $h \equiv\left(2 A_{t}\right) / p_{t}$. The definition of $\beta_{i}$ and Eq. (6) yields a nonlinear equation to find $L^{*}$. All the lengths appearing on the problem are scaled with this length

$$
\xi=\frac{x}{L^{*}} \in\left[0, \ell_{t}\right]
$$

where $\ell_{t}=L / L^{*}$.

The characteristic current is the short circuit current, i.e., $I_{s c}=\sigma E_{m} A_{t}$. Conversely, the bias due to the induced electric field along the characteristic length, $E_{m} L^{*}$, is used as the characteristic voltage drop. Finally, the dependent nondimensional variables $i$ and $\varphi$ are defined as

$$
\begin{gathered}
i(\xi)=I / I_{s c} \\
\varphi(\xi)=\Phi /\left(E_{m} L^{*}\right)
\end{gathered}
$$

The nondimensional form of the parameters related to the electric devices is

$$
\begin{gathered}
\tilde{V}_{c c}=\frac{V_{c c}}{E_{m} L} \\
\Omega=\frac{r \sigma A_{t}}{L}
\end{gathered}
$$

This paper will use subscripts $A, B$, and $C$ in the variables $\varphi$ and $i$ to denote the values of these magnitudes for special points along the tether (Fig. 1).

The nondimensional form of the system of differential equations and boundary conditions for passive tethers is presented next.

\section{Anodic Segment}

Bias and current profile are governed by Eqs. (2) and (4)

$$
\begin{gathered}
\frac{d i}{d \xi}=\frac{3 G\left(\beta_{i} \varphi ; T_{e} / T_{i}, R / \lambda_{D i}\right)}{4\left(\beta_{i} ; T_{e} / T_{i}, R / \lambda_{D i}\right)} \sqrt{\varphi} \approx \frac{3}{4} \sqrt{\varphi} \\
\frac{d \varphi}{d \xi}=i-1
\end{gathered}
$$

where the ratio $G\left(\beta_{i} \varphi ; T_{e} / T_{i}, R / \lambda_{D i}\right) / G\left(\beta_{i} ; T_{e} / T_{i}, R / \lambda_{D i}\right)$ was approximated to 1 because the dependence of $G$ with the bias is very weak (Estes and Sanmartín 2000) and it can be safely ignored for large potentials (G. Sanchez-Arriaga et al., "Impact of non ideal effects on bare electrodynamic tether performance," submitted, ETSIA, Madrid, Spain). The boundary conditions are

$$
\begin{gathered}
\xi=0: i=0 \\
\varphi=0: i=i_{B}
\end{gathered}
$$

where $i_{B}$ is an unknown to be determined with the current profile solution.

\section{Cathodic Segment}

Bias and current profile are governed by Eqs. (3) and (4). This set of ordinary differential equations of the cathodic segment can be identical to the ones of the anodic segment, providing that an appropriate set of variables is used

$$
\eta \equiv-\mu^{2 / 3}\left(\xi-\xi_{f}\right)
$$

where

$$
\begin{gathered}
\mu \equiv\left(\frac{m_{e}}{m_{i}}\right)^{1 / 2} \frac{G\left(\beta_{e}, T_{e} / T_{i}, R / \lambda_{D e}\right)}{G\left(\beta_{i}, T_{e} / T_{i}, R / \lambda_{D i}\right)} \\
\psi \equiv-\mu^{2 / 3} \varphi
\end{gathered}
$$

Eqs. (3) and (4) read

$$
\begin{aligned}
& \frac{d i}{d \eta} \approx \frac{3}{4} \sqrt{\psi} \\
& \frac{d \psi}{d \eta}=i-1
\end{aligned}
$$

where it has been assumed $G\left(\beta_{e} \varphi ; T_{i} / T_{e}, R / \lambda_{D e}\right) / G\left(\beta_{e} ; T_{i} /\right.$ $\left.T_{e}, R / \lambda_{D e}\right) \approx 1$. Choosing $\xi_{f}$ as

$$
\xi_{f}=\xi_{B}\left(1+\frac{1}{\mu^{2 / 3}}\right)
$$

one has $\eta=0$ and $\eta=\xi_{B}$, if $\xi=\xi_{f}$ and $\xi=\xi_{B}$, respectively. With the new variables, the solution is symmetric with respect to the point of zero bias (Fig. 2). The boundary condition of the cathodic segment at Point $\mathbf{B}$ then reads

$$
\eta=\xi_{B}: \psi=0, \quad i=i_{B}
$$

and Eqs. (17) and (18) are formally identical to Eqs. (10) and (11). Without any contactor at the cathodic end, the current vanishes at both ends and the solution corresponds to a floating tether of length $\xi_{f}(\eta=0)$. When a contactor is present, the last boundary condition required to close the problem is the circuit equation, as depicted in Fig. 2. Circuit Eq. (5) in nondimensional variables reads

$$
\left\{\left[\left(\Omega i_{C}\right)+\tilde{V}_{c c}\right] \ell_{t} \mu^{2 / 3}\right\}-\psi_{C}=0
$$

Ignoring the ratios of the $G$ functions in Eqs. 10 and 16 simplifies the problem notably (G. Sanchez-Arriaga et al., "Impact of non ideal effects on bare electrodynamic tether performance,"

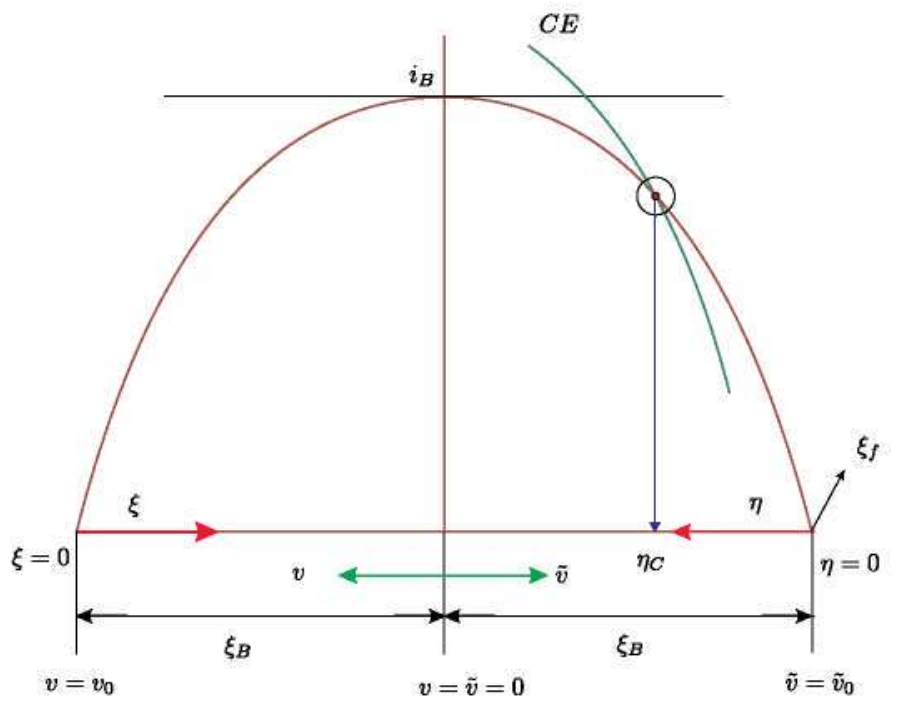

Fig. 2. Symmetry of the anodic and cathodic description for the proposed change of variables; label CE represents the boundary condition of the circuit equation in the $\eta$ - $i$ semiplane 
submitted, ETSIA, Madrid, Spain). The beyond OML effect is incorporated in $L^{*}$, which is a factor $1 /\left(G^{2 / 3}\right)$ larger as compared with the OML regime, and the plasma parameters $T_{e} / T_{i}$, $R / \lambda_{\text {De }}$, and $\left(e E_{m} L_{*}\right) /\left(k T_{e}\right)$ do not affect the dimensionless equations governing the current and potential profiles.

\section{Semianalytical Solution}

The systems of Eqs. (10), (11), (17), and (18) are autonomous. The vector fields in the state spaces $(\varphi, i)$ and $(\psi, i)$ have the form

$$
\begin{aligned}
& \frac{d i}{d \varphi}=\frac{-\frac{3}{4} \sqrt{\varphi}}{1-i} \\
& \frac{d i}{d \psi}=\frac{-\frac{3}{4} \sqrt{\psi}}{1-i}
\end{aligned}
$$

The family of solutions of the differential Eqs. (22) and (23) can be expressed analytically

$$
\begin{aligned}
& \varphi\left(i ; i_{B}\right)=\left(i_{B}-i\right)^{2 / 3}\left(2-i_{B}-i\right)^{2 / 3} \\
& \psi\left(i ; i_{B}\right)=\left(i_{B}-i\right)^{2 / 3}\left(2-i_{B}-i\right)^{2 / 3}
\end{aligned}
$$

where $i_{B}$ is the parameter that determines the particular solution of the family. A representation of the solutions in the state plane $(\varphi, i)$ can be found in Fig. 3. The boundary conditions and the operational physic limits can also be represented in the state plane. The circuit Eq. (21) corresponds to a straight line. The physical limit of not exceeding the short circuit equation corresponds to a horizontal line at $i=1$. The solution of the problem is in accordance with the orbit among the possible trajectories in the state plane, which fulfills that in the intersection with the boundary conditions, the variable $\xi$ is equal to $\ell_{t}$ [or $\eta$ is equal to $-\mu^{2 / 3}\left(\ell_{t}-\xi_{f}\right)$ ]. There is a singular point in the state space, i.e., $\varphi=\psi=0, i=1$. The equilibrium solution corresponds to zero bias and short-circuit current along an arbitrary length of the tether, $\xi_{S}$ in nondimensional variables. The value of $\xi_{S}$ can be determined as part of the solution when the boundary conditions are imposed.

The relation between current and tether location in the anodic segment (similarly for the cathodic) is given by

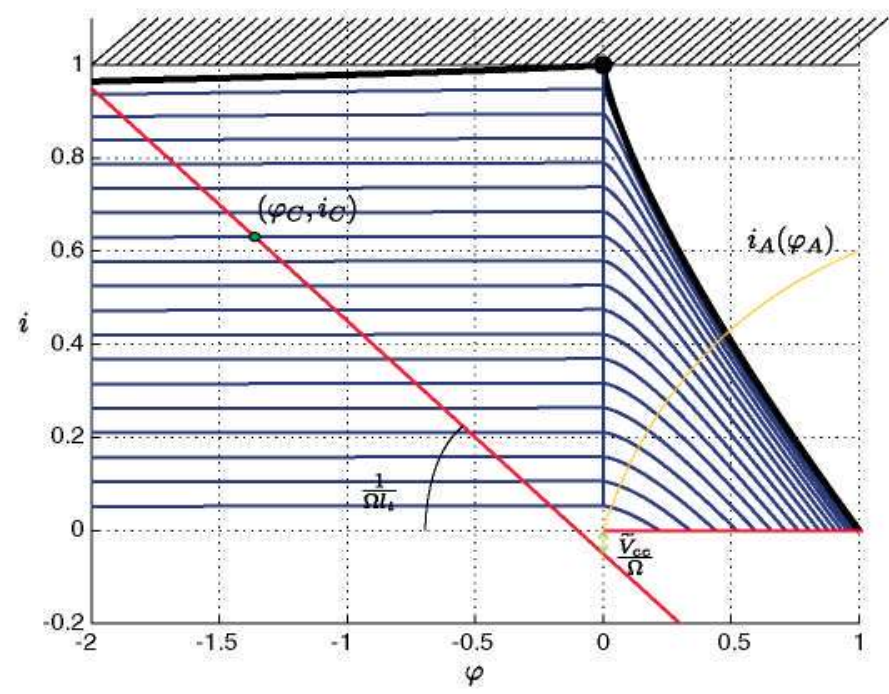

Fig. 3. Solutions in the phase plane

$$
\xi=\frac{4}{3} \int_{0}^{i} \frac{d \zeta}{\left(i_{B}-\zeta\right)^{1 / 3}\left(2-i_{B}-\zeta\right)^{1 / 3}}
$$

At this point, two auxiliary variables, (1) $v$ in the anodic segment, and (2) $\tilde{v}$ in the cathodic segment, are defined (Fig. 2). The introduction of $v$ allows researchers to obtain an explicit parametric expression of $i$ and $\xi$. In the anodic segment, the parametric description is

$$
\begin{gathered}
\varphi(i)=\left(i_{B}-i\right)^{2 / 3}\left(2-i_{B}-i\right)^{2 / 3} \\
i(v)=1-\left[\left(1-i_{B}\right) \cosh (v)\right] \\
\xi(v)=\frac{4}{3}\left[\left(1-i_{B}\right)^{1 / 3}\right]\left[f\left(v_{0}\right)-f(v)\right]
\end{gathered}
$$

where $v \in\left[0, v_{0}\right]$. As it can be seen in Fig. 2, $v=v_{0}$ at the anodic end and $v=0$ at Point $\mathrm{B}$. The value of $v_{0}$ can be expressed as a function of the parameter of the family of solutions $i_{B}, v_{0}=$ $\cosh ^{-1}\left[1 /\left(1-i_{B}\right)\right]$. In the parametric representation of $\xi$, an integral function, $f(x)$, comes up. It is defined as

$$
f(x)=\int_{0}^{x} \sinh ^{1 / 3}(\zeta) d \zeta
$$

The efficient evaluation of $f(x)$ is crucial and is discussed in the subsequent text. The previous description is valid for $i_{B} \neq 1$. When $i_{B} \equiv 1$ the parametric description turns out to be

$$
\begin{gathered}
\varphi(i)=(1-i)^{4 / 3} \\
\xi(i)=4\left\{1-\left[(1-i)^{1 / 3}\right]\right\}
\end{gathered}
$$

with $i \in[0,1]$. The approach on the cathodic segment is analogous and Eqs. (27), (29), and (31) are valid, changing $\varphi \rightarrow \psi, \xi \rightarrow \eta$, and $v \rightarrow \tilde{v}$. The difference lies in the boundary conditions and thus $\tilde{v}$ varies between $\left[0, \tilde{v}_{C}\right]$. The unknown of the problem is $\tilde{v}_{C}$ and should fulfill

$$
\eta\left(\tilde{v}_{C}\right)=\xi_{B}-\left[\mu^{2 / 3}\left(\ell_{t}-\xi_{B}\right)\right]
$$

The circuit [Eq. (20)] should also be fulfilled.

As previously indicated, the efficiency in the computation of the nondimensional current and bias profiles depends on the ability to produce a fast evaluation of the function $f(x)$, defined previously. There exists an analytical solution for $f(x)$ in terms of hypergeometric functions. However, in order to speed up the calculation, $f(x)$ is computed using an asymptotic formulation and a series expansion. Both formulations are found with the help of algebraic manipulators. The behavior of $f(x)$ when $x \rightarrow \infty$ is given by

$$
\begin{aligned}
f(x) \asymp & {\left[3\left(\cosh ^{1 / 3} x\right)\right]-k_{1}-\left[\frac{1}{5}\left(\cosh ^{-5 / 3} x\right)\right]-\left[\frac{2}{33}\left(\cosh ^{-11 / 3} x\right)\right] } \\
& -\left[\frac{14}{459}\left(\cosh ^{-17 / 3} x\right)\right]+\left[\mathcal{O}\left(\cosh ^{-17 / 3}\right)\right]
\end{aligned}
$$

where constant $k_{1}$ has the value

$$
k_{1}=\frac{1}{\pi} 2^{-2 / 3} 3^{3 / 2}[\Gamma(2 / 3)]^{3} \approx 2.5871
$$

The relative error of this asymptotic approximation is below $10^{-10}$ for $x \geq 3$. Conversely, a power series expansion of $f(x)$ is used for computing the value of the function for $x<3$. For completeness, the expansion is gathered 


$$
\begin{gathered}
f(x) \asymp x^{4 / 3}\left[\frac{3}{4}+\left(\frac{1}{60} x^{2}\right)-\left(\frac{1}{17,280} x^{4}\right)+\left(\frac{53}{8.98128 \times 10^{6}} x^{6}\right)\right. \\
\left.-\left(\frac{191}{5.878656 \times 10^{8}} x^{8}\right)\right]+\left[\mathcal{O}\left(x^{28 / 3}\right)\right]
\end{gathered}
$$

Although the convergence of the series expansion is not fast, the number of terms can be extended with no harm to the computation performance.

\section{Computational Algorithm}

The steps to obtain the current and Lorentz force at a given instant of time are described in detail in this section. The data available at the beginning of the computation involve tether parameters, including $L$, cross-sectional dimensions ( $R$ for round tethers and $w$ and $h$ for tape tethers), and $V_{c c}, r$, and $\sigma$ and environmental variables, i.e., $E_{m}, N_{0}, T_{t}$, and $T_{i}$. The nondimensional length of the tether $L^{*}$ can then be computed using the function $G$ as it is described in the Appendix. Researchers can easily work out the value $L^{+}$thanks to the assumption that the dependence of $G$ on its first argument is negligible. The other characteristic and derived magnitudes are also found. They allow researchers to obtain the nondimensional parameters $\ell_{f}, \Omega$, and $\tilde{V}_{c c}$. These parameters determine the boundary conditions through Eq. (21).

The parametric representation given by Eqs. (27) and (29) has two unknown parameters, as follows: $(1) i_{B}$ (which selects the orbit of the family of solutions), and (2) $i_{C}$ (which determines the arc length corresponding to the nondimensional length of the tether $\left.\ell_{i}\right)$. The solution must satisfy the constraints of Eqs. (21) and (33). Therefore, the problem is closed and it is well-posed within the allowable range of parameters. For a more compact formulation of the algorithm, the variable $\delta$ is used instead of $i_{C}$, i.e., $i_{C}=$ $i_{B}-\delta$. Substituting the previous into Eqs. (21) and (33), a system of two equations [i.e., (1) Eq. (37), and (2) Eq. (38)] with two unknowns [i.e., (1) $\delta$, and (2) $i_{B}$ ] is obtained

$$
\begin{gathered}
\mu^{2 / 3} \ell_{t}=\frac{4}{3}\left[\left(1-i_{B}\right)^{1 / 3}\right]\left\{\mu^{2 / 3}\left[f\left(v_{\theta}\right)+f\left(\tilde{v}_{C}\right)\right]\right\} \\
\mu^{2 / 3}\left[\left(\Omega \ell_{t} i_{B}\right)+\left(\tilde{V}_{c \tau} \ell_{t}\right)\right]=\left(\mu^{2 / 3} \Omega \ell_{t} \delta\right) \\
+\left[\left(\delta^{2 / 3}\right)\left(\left\{\left[2\left(1-i_{B}\right)\right]+\delta\right\}^{2 / 3}\right)\right]
\end{gathered}
$$

where $\tilde{v}_{C}=\cosh ^{-1}\left\{1+\left[\delta /\left(1-i_{B}\right)\right]\right\}$.

In this way, the boundary value problem is formulated as finding the root of a two-dimensional nonlinear function of two variables [i.e., (1) $i_{B}$, and (2) $\delta$ ]. Therefore, a conventional zero-finding computational algorithms can be used in order to solve for the unknowns. This formulation, using $\delta$ instead of $i_{C}$ or $\tilde{v}_{C}$ as the unknown, presents two main advantages, as follows:

1. $\delta \ll 1$ because the current drop along the cathodic segment is small for common tether lengths and electric loads, and this allows researchers to generate a good guess in the iterative process

$$
\delta_{0} \approx \frac{\mu\left[\left(\Omega \ell_{t} i_{B}\right)+\left(\tilde{V}_{c c} \ell_{t}\right)\right]^{3 / 2}}{2\left(1-i_{B}\right)}
$$

This approximation is not valid if $i_{B} \equiv 1$. In such a case, $\delta \approx \mu^{1 / 2}\left[\ell_{s}\left(\Omega+\tilde{V}_{c c}\right)\right]^{3 / 4}$.

2. The equation in $\delta$ (with $i_{B}$ as the parameter), i.e., Eq. (38), is simpler than the equation in $\tilde{v}_{C}$ (with $i_{B}$ as the parameter), i.e., Eq. (37). Therefore, efficient methods for searching roots can be used, such as the Newton Raphson method.

\section{Validation and Comparison}

The validation of the work presented in this paper is twofold, as follows: (1) the correction to the OML regime is compared with the results of Estes and Sanmartín (2000) and Sanmartín and Estes (1999), and (2) the computational algorithm is compared to prior solutions found in the literature. Concerning the former, the formulas which are introduced in the Appendix provide $G$ with an error below a few percent in common tether operation [as compared with the results from Estes and Sanmartín (2000)]. In the worst cases, which involve very extreme conditions, the error is below $8 \%$. Taking into account the uncertainties in the environmental parameters and the assumption made in different part of the analysis (high bias approximation, straight tether, and constant tether temperature and conductivity), this is an acceptable error. The alternative, i.e., the exact solutions of the shooting problem posed in Estes and Sanmartín (2000), would slow down the tether flight simulator.

Regarding the latter, three methods have been implemented for the passive regime, whereas two methods were developed for the active regime. All the methods are intended to solve the problem formulated in the nondimensional form. Therefore, the solution consists of the pair $i_{B}, \delta\left(i_{B}, i_{C}\right)$ given the input parameters $\ell_{t}, \Omega$, $\tilde{V}_{c c}$, and $\mu$. The input parameters are fed randomly (although all the methods solve for the same case); the values are taken from a continuous uniform distribution.

A shooting method is used as benchmark. The algorithm is based on a shooting method to a fitting point according to Section 18.2 of Press et al. (1992). The integration of the equations of motion is made for anode and cathode independently, starting at both ends of the tether. The matching of the solution is imposed at Point $\mathbf{B}$. This is a suitable method because of the possible singular solution $i=1, \varphi=0$ at Point B. Moreover, a method based on the utilization of hypergeometric functions as described in Leamy et al. (2001) has been derived for the passive regime.

The validation is conducted in terms of the relative and absolute difference of the proposed algorithm with respect to the reference method (shooting). Regarding the relative error, a batch of computations has been carried out for random values of the parameters $\ell_{t}$ and $\Omega$. To perform the comparison, the integral of the current along the tether, $\int_{0}^{t_{i}} i(\xi) d \xi$, has been considered. The results show that almost all the cases are below a relative enror of $10^{-3}$. Those which are above that threshold correspond to either values of (1) $\ell_{t} \ll 1$, or (2) the region of the parameter space where $\left(i_{B}=1, \phi_{B}=0\right.$ ) along $\xi_{S}$. In the first situation, the integral of the current is too small and therefore the relative errors increase. Nevertheless, the absolute error remains bounded. In the second case, the problem lies in the

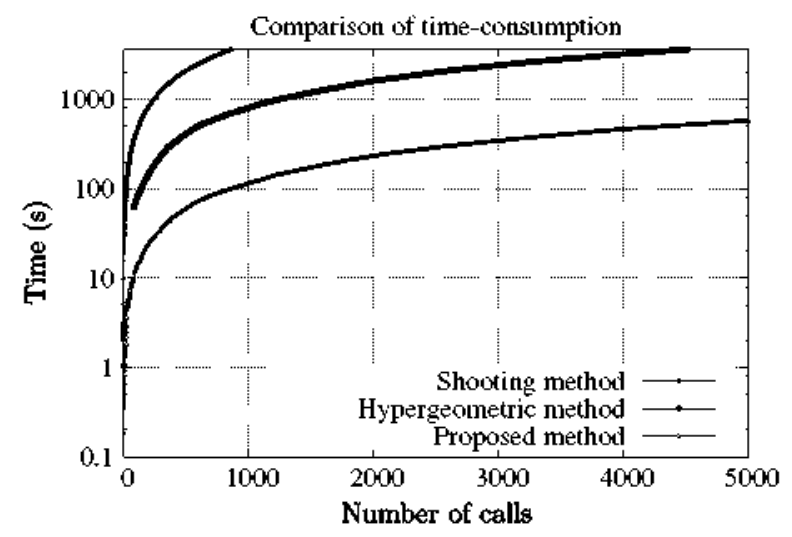

Fig. 4. Computational time in seconds versus number of calls for each method 
difficulty of the shooting method to produce an accurate solution when the singular solution $i=1, \varphi=0$ is present along a segment.

In addition, a comparison of the performance is made in terms of the computational time. In Fig. 4, this comparison is presented. The semianalytical method introduced in this paper is about one order of magnitude faster than the method based on hypergeometric functions and two orders of magnitude more rapid than the conventional shooting method.

\section{Conclusion}

This paper addresses the fast and accurate computation of the current along a bare electrodynamic tether, for variable environmental and dynamical conditions. This is mandatory for the simulation of bare EDTs dynamics and operation, and for the assessment of its performance. A semianalytical approach is derived to satisfy the computational requirements in terms of time consumption using a state-of-the-art current collection model.

The approach is based on the use of a change of variables that reduces the two-dimensional two-point boundary value problem to a two-dimensional root-finding problem. The latter is solved sequentially in two steps, using at each step (1) conventional onedimensional root-finding algorithms as bisection, or (2) the Newton Rapson methods.

The numerical comparison between the proposed method and those found in the literature finds a good agreement in the vast majority of cases. The lack of agreement takes place in special situations in which the shooting method seems to be unable to find the profile solution. Finally, the results of the proposed method show an important time-consumption improvement with respect to the previous methods.

The beyond OML effect was incorporated in the model with a very low computational cost. This can be useful for certain missions, which must be carried out with wide tethers to have a small cut probability (Khan and Sanmartin 2013). Thanks to the proposed fitting, the algorithm just needs to evaluate the analytical function $G$ at each time step to find the correct value of $L^{*}$. In any case, beyond OML effect is not expected to have a strong impact on the deorbit time because (1) function $G$ does not decay very fast with the ratio $R / \lambda_{D e}$, and (2) $R$ is typically beyond $R_{\max }$ just for low altitudes (where plasma density is higher and the Debye length is smaller, and the tether spends a small fraction of time there). However, although the Lorentz force computed with and without the beyond OML effect can be close to each other at certain time steps, it is a cumulative effect that may affect tether behavior. For instance, a self-balanced tether mitigates the dynamic instability because a dimensionless parameter involving the Lorentz torque about the center of mass, say $\epsilon$, is very small (Peláez and Sanjurjo 2006). Since this torque is affected by the current profile, small variations like the one produced secularly by the beyond OML effect, can produce a nonnegligible effect [the growth rate varies as $\epsilon^{3}$ (Peláez et al. 2000)].

There are some limitations of the model that should be taken into account. The high bias hypothesis $[(e \Phi) /(k T) \gg 1]$, underlies most of the analysis, including the determination of the function $G$, the equivalent radius rule $\left(R_{\mathrm{eq}}=w / 4\right)$ for tape tethers and the OML law itself, which has a term with a complementary error function that was ignored in this paper. Plasma thermal energy, about $0.15 \mathrm{eV}$, typical tether lengths, and motional electric field values normally meet the requirement $(e \Phi) /(k T) \gg 1$. However, for a tether orbiting in the $\mathrm{F}$ layer, where $\mathrm{O}^{+}$is the dominant ion species, ion (ram) energy is large compared with the thermal energy and a paradox appear in stationary Langmuir probe theories
(Sanmartín 2002). Recent simulations showed the following: (1) the paradox is explained if electron trapping is included, and (2) collected current is not affected severely by the ram effect (Sánchez-Arriaga and Pastor-Moreno 2014). At higher altitudes, where $\mathrm{H}^{+}$is dominant, the ram effect can be ignored. Regarding the tape tether, potential barriers always appear and OML current is not achieved; current reduction below the OML value is of the order $\left(1 /\left\{\ln \left[(e \Phi) /\left(k T_{i}\right)\right]\right\}\right)^{2}$ as per Sanmartín and Estes (1999).

\section{Appendix. Correction to the OML Regime}

The right-hand sides in Eqs. (2) and (3) involve the functions $G$, which takes into account the formation of potential barriers at $R$ and the deviation of the current from the OML regime. This function was computed in Estes and Sanmartín (2000) for a cylindrical probe of radius $R$ in the high and positive bias case $e \Phi \gg k T_{e}$. The probe is considered immersed at rest in an unmagnetized equilibrium plasma with electron and ion temperatures $T_{e}$ and $T_{i}$, respectively. Its determination involves the solution of a boundary value problem, which is cumbersome for tether flight simulators. In this paper a simple analytical fitting is proposed that allows the inclusion of the beyond OML effect without a significant increase in the computational cost. For tape tethers, one may take $R \approx w / 4$, where $w$ is the width of the tape (Sanmartín and Estes 1999).

Function $G(\chi, \mu, \rho)$ has arguments $\chi, \mu$, and $\rho$, which involves the normalized bias, the temperature ratio, and the normalized probe radius, respectively. As shown in Sanmartín and Estes (1999), there is a maximum normalized radius of the probe $\rho_{\max }$ to operate within the OML regime. Therefore, if $\rho<\rho_{\max }$ one has $G=1$. A simple fitting of $\rho_{\max }$ obtained from the high bias results of Sanmartín and Estes (1999) is

$$
\rho_{\max }(y \equiv \ln \chi, \mu)=\frac{\left(p_{1} y^{2}\right)+\left(p_{2} y\right)+p_{3}}{y+p_{4}}
$$

where

$$
\begin{gathered}
p_{1}=\frac{(0.08 \mu)+0.11}{\mu+0.19} \\
p_{2}=\frac{(-0.34 \mu)-0.26}{\mu+0.065} \\
p_{3}=\frac{(1.63 \mu)+1.73}{\mu+0.11} \\
p_{4}=\frac{\left(1.8 \mu^{2}\right)-(0.48 \mu)+0.22}{\mu+0.3}
\end{gathered}
$$

If $\rho>\rho_{\max }$, the tether operates beyond the OML regime and $G$ drops below 1. In Estes and Sanmartín (2000) it was shown that the dependence on probe bias is very weak and one can write $G=G\left(\mu, \rho-\rho_{\max }\right)$. A fitting to the results obtained in Estes and Sanmartín (2000) at the particular case $\chi=1000$ is

$$
G\left(\mu, z \equiv \frac{\rho-\rho_{\max }}{\sqrt{\mu}}\right)=\frac{\left(c_{1} z^{2}\right)+\left(c_{2} z\right)+c_{3}}{z^{2}+\left(c_{4} z\right)+c_{3}}
$$

where 


$$
\begin{gathered}
c_{1}=\frac{\left(0.19 \mu^{2}\right)+(0.056 \mu)+0.0182}{\mu^{2}+(0.95 \mu)+0.26} \\
c_{2}=\frac{(1.46 \mu)+1.29}{\mu+0.045} \\
c_{3}=\frac{(17.2 \mu)-0.82}{\mu+0.13} \\
c_{4}=\frac{(1.56 \mu)+1.12}{\mu+0.037}
\end{gathered}
$$

\section{Acknowledgments}

The work of M. Sanjurjo-Rivo and J. Peláez is part of the research project entitled Dynamic simulation of complex space systems (AYA2010-18796) supported by the Spanish Ministry of Economy and Competitiveness. The writers thank the Spanish Government for its financial support. The work of G. Sánchez-Arriaga is part of the FP7/Space Project 262972 supported by the European Commission.

\section{Notation}

The following symbols are used in this paper:

$$
\begin{aligned}
A_{t} & =\text { tether transverse area; } \\
\mathbf{B}_{0} & =\text { geomagnetic field; } \\
\mathbf{E} & =\text { electric field; } \\
e & =\text { electron charge; } \\
I & =\text { current along the tether; } \\
i & =\text { nondimensional current; }
\end{aligned}
$$

$I_{\mathrm{SC}}=$ short circuit tether current;

$L=$ tether length;

$L^{*}=$ characteristic tether length;

$\ell_{t}=$ nondimensional tether length;

$m_{e}=$ electron mass;

$m_{i}=$ ion mass;

$N_{0}=$ density of the ionospheric plasma;

$p_{t}=$ perimeter of the tether;

$R$ = radius of a round tether;

$r=$ load resistance;

$T_{e}=$ electron temperature;

$T_{i}=$ ion temperature;

$\mathbf{u}=$ unit vector from cathodic to anodic end;

$V_{c c}=$ potential bias of cathodic contactor;

$\tilde{V}_{c c}=$ nondimensional potential bias of cathodic contactor;

$V_{p l}=$ plasma potential;

$\mathbf{v}_{\text {rel }}=$ tether-to-plasma relative velocity;

$V_{t}=$ tether potential;

$w=$ width of a tape tether;

$x=$ coordinate along the tether;

$\epsilon=$ potential bias of power generator;

$\Phi=$ potential bias between conductor and faraway plasma;

$$
\begin{aligned}
\sigma & =\text { tether conductivity; } \\
\varphi & =\text { nondimensional potential bias; } \\
\Omega & =\text { nondimensional electrical load; and } \\
\xi & =\text { nondimensional arc tether length. }
\end{aligned}
$$

\section{References}

Ahedo, E., and Sanmartín, J. R. (2002). "Analysis of bare-tether systems for deorbiting low-earth-orbit satellites." J. Spacecraft Rockets, 39(2), 198-205.

Bombardelli, C., Peláez, J., and Sanjurjo, M. (2010). "Asymptotic solution for the current profile of passive bare electrodynamic tethers." J. Propul. Power, 26(6), 1291-1304.

Estes, R. D., and Sanmartín, J. R. (2000). "Cylindrical Langmuir probes beyond the orbital-motion-limited regime." Phys. Plasmas, 7(10), $4320-4325$

Johnson, L., Estes, R. D., Lorenzini, E., Martínez-Sánchez, M., and Sanmartín, J. (2000). "Propulsive small expendable deployer system experiment." J. Spacecraft Rockets, 37(2), 173-176.

Khan, S. B., and Sanmartín, J. R. (2013). "Survival probability of round and tape tethers against debris impact." J. Spacecraft Rockets, 50(3), 603-608.

Laframboise, J. G., and Parker, L. W. (1973). "Probe design for orbitlimited current collection." Phys. Fluids, 16(5), 629-636.

Leamy, M., Noor, A., and Wasfy, T. (2001). "Sensitivity analysis of bare-wire space tether systems," Comput. Methods Appl. Mech. Eng., 190(42), 5495-5503.

Peláez, J., Lorenzini, E. C., López-Rebollal, O., and Ruiz, M. (2000). "A new kind of dynamic instability in electrodynamic tethers." Adv. Astronaut. Sci., 105, 1367-1386.

Peláez, J., and Sanjurjo, M. (2006). "Generator regime of self balanced electrodynamic tethers." J. Spacecraft Rockets, 43(6), 1359-1369.

Press, W. H., Teukolsky, S. A., Vetterling, W. T., and Flannery, B. P. (1992). Numerical recipes in C: The art of scientific computing, 2 nd Ed., Cambridge University Press, Cambridge.

Sánchez-Arriaga, G., and Pastor-Moreno, D. (2014). "Direct Vlasov simulations of electron-attracting cylindrical Langmuir probes in flowing plasmas." Phys. Plasmas, 21(7), 073504.

Sánchez-Torres, A. (2013). "Electrodynamic tethers for planetary and de-orbiting missions." Ph.D. thesis, Univ. Politécnica de Madrid, Madrid, Spain.

Sanmartín, J. R. (2002). "Active charging control and tethers." CNES-space technology course: Prevention of risks related to spacecraft charging, J. P. Catani, ed., Cepadus, Toulouse, France, 515-533.

Sanmartín, J. R., et al. (2006a). "Floating bare tether as upper atmosphere probe." J. Geophys. Res., A11, A11310.

Sanmartín, J. R., et al. (2010). "Electrodynamic tether applications and constraints." J. Spacecraft Rockets, 47(3), 442-456.

Sanmartín, J. R., Elaskar, S., Estes, R., and Lorenzini, E. (2006b). "Efficiency of electrodynamic tether thusters." J. Spacecraft Rockets, 43(3), 659-666.

Sanmartín, J. R., and Estes, R. (1999). "The orbital-motion-limited regime of cylindrical Langmuir probes." Phys. Plasmas, 6(1), 395-405.

Sanmartín, J. R., Estes, R., and Lorenzini, E., (2001). "Efficiency of different types of ED-tether thrusters." Proc., Space Technology and Applications International Forum (STAIF), American Institute of Physics, Melville, NY, 479-487.

Sanmartín, J. R., and Lorenzini, E. (2005). "Exploration of the outer planets using tethers for power and propulsion." J. Propul. Power, 21(3), 573-576.

Sanmartín, J. R., Martínez-Sánchez, M., and Ahedo, E. (1993). "Bare wire anodes for electrodynamic tethers." J. Propul. Power, 9(3), 353-360. 